\title{
Frequency selective surfaces on flexible thin plastic substrates
}

\author{
N. A. F. M. Zainudin, S.N. Azemi, M.F.Jamlos, A.A.Al-Hadi, P.J. Soh \\ Advanced Communication Engineering Center (ACE), School of Computer and Communication Engineering, \\ University Malaysia Perlis, Malaysia
}

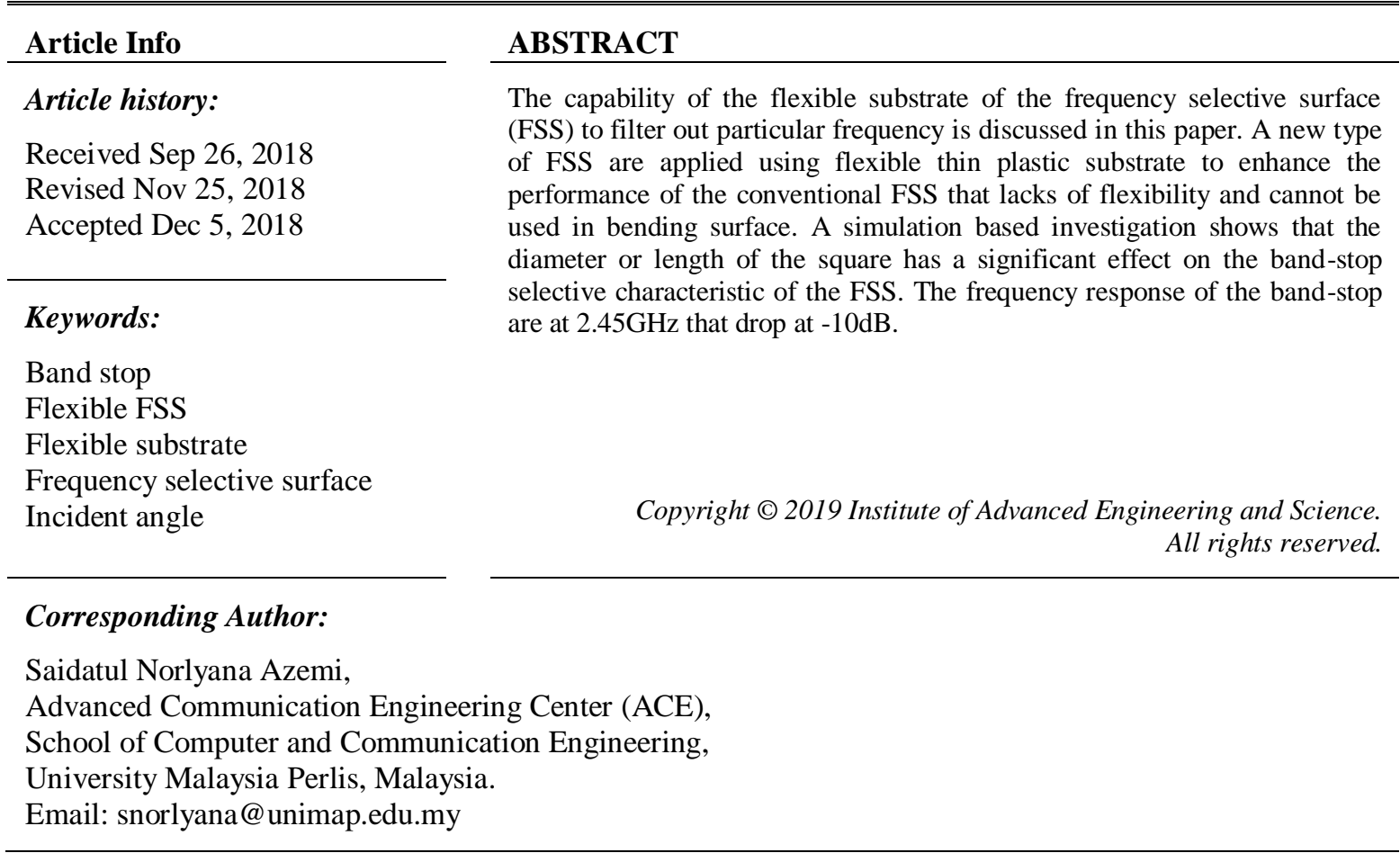

\section{INTRODUCTION}

Requency Selective Surfaces (FSS), have been known for a long time around since 50 years ago. Ben Munk [1], is the one who probably introduced and guided the practical design for the FSS. FSS usually are formed by periodic array of radiating elements which effectively act as filter correspondingly to electromagnetic waves. Generally frequency-selective surface is a 2D planar structure consisting of one or more metallic patterns, each backed by a dielectric substrate. These structures are usually arranged in a periodic fashion and their frequency response is entirely determined by the geometry of the structure in one period called a unit cell. There are a wide variety of possible elements which can be used to realize FSS such as round shape, square shape, fractal shape and many more. Different element shapes offer FSS different frequency responses. Depending on their physical construction, material and geometry, they are divided into four categories which are low-pass, high-pass, band-pass and band-stop filters [1]-[2].

This elements and slots of arrays effectively creates band stop and band pass filters respectively to electromagnetic energy at their designated frequency range. A classical FSS consists of a two dimensional periodic array of certain shapes that are either printed on a dielectric substrate or etched through a conductive layer. It is been commonly used as an electromagnetic shielding. Recently, applications like selective frequency shielding of room windows, in train and aircraft windows to isolate the unwanted radiation and antenna radomes, have emerged in the past decade. It has increased the demand of a FSS on a dielectric which is optically transparent and also flexible [3]-[6]. However, there is deficiency in research and implementation of the flexible and transparent FSS. Polycarbonate is one of the types of clear optical plastic with refractive index of 1.6 and wide range of temperature and chemical resistance. It is a suitable substrate for the analysis which can provide both clarity and flexibility [7]-[10]. However, the ability as a substrate yet need to be evaluated. In this paper a square elements shape FSS is proposed. The investigation on the structure of the single layer FSS of the square loop elements are perform and the substrate are change 
according to the substrate bring used. The length and the size of the square are varying according to the design proposed. By varying the size of the unit cell size and the length of the inner and outer square, the frequency response are adjusted and a closer band stop response are achieved. The parametric analysis of the square elements shape FSS on the flexible thin plastic substrate are undertaken using the CST software and experimental validation is underway.

\section{FREQUENCY SELECTIVE SURFACE SQUARE DESIGN}

The equivalent circuit and the basic design of the square elements shape of FSS for this paper are shown in Figure 1. The square denotes the presence of the conductor material which is copper. However, the square width must be maintain at very thin to ensure the substrate flexibility. The conducting square elements shape are self-inductance due to loop of the charge flow and also a mutual inductance due to coupling with the adjacent loops. The charge distribution across the small gap between the adjacent square elements shape creates the substantial capacitance. When the cascading capacitance and induction structure are combine together it will produces a low impedance surface near its resonant frequency, producing a stop band that blocked the transmission wave through the FSS structures. The lumped elements of the LC equivalent circuit suggest that, when the gap width s between two adjacent square elements shape decrease, it will increase the capacitance value and will reduces the stop band frequency [12]-[13]. Also increase the inner length of the elements shape will decrease the width track and increase the current flow path, this will increase the inductance and decrease the stop band frequency. So, smaller length of the square elements shape result in higher frequency response and smaller unit cell size will ensure good frequency response with vary incident angles [14]-[16].

The FSS parameters of the square elements shape are obtain from equation:

$$
\begin{aligned}
& \lambda=\frac{c}{f} \\
& d 1=\frac{\lambda}{\pi} \\
& d 2=\frac{d 1}{\lambda}
\end{aligned}
$$

In the equation (1), the bandwidth, $\lambda$ can be determine from the value of the speed of light, $c$ and the frequency response, $f=2.45 \mathrm{GHz}$. From the obtain bandwidth the value of the outer square length, $d 1$ and length of the inner square, $d 2$ can be determine. The fabrication process of this paper are done manually by spray the silver ink using the air brush on the plastic substrate. The fabricated FSS on flexible thin plastic substrate are shown in Figure 2.

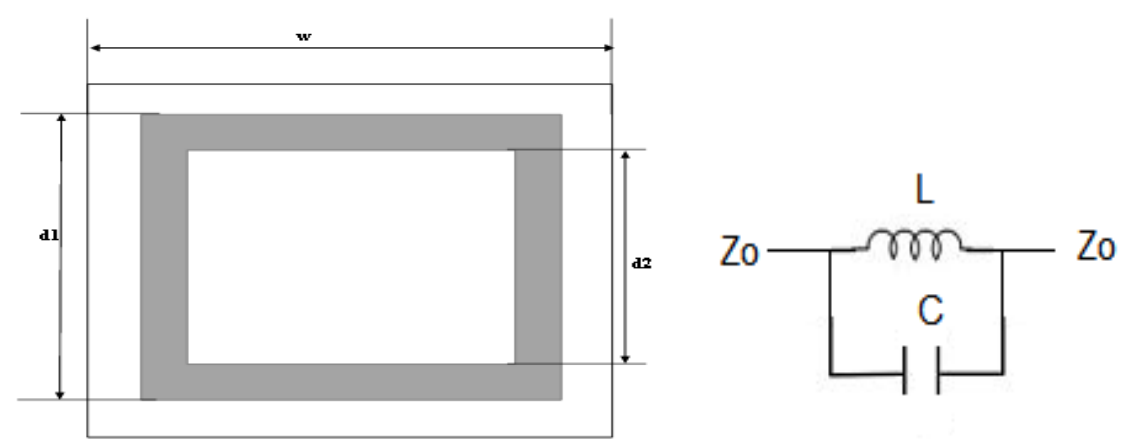

Figure 1. Square FSS elements shape and equivalent circuit

\section{RESULT AND DISCUSSION}

The simulation results for the FSS on the flexible thin plastic substrate design based on the parametric study done be done. A 2-D square elements shape is used in this project just as shown in Figure 3. As the primary interest for this paper is the S-parameter results, which represent the transmission and reflection for this FSS. The co-polar reflections and transmission for both modes are almost identical due to the symmetrical square elements shape. As shown in the Figure 4, the transmission of the FSS is almost 
completely blocked at the frequency drop at $2.45 \mathrm{GHz}$, as seen from the $\mathrm{S} 21$ is about $-65 \mathrm{~dB}$, and the reflection is almost complete $\mathrm{S} 11 \approx-0.004 \mathrm{~dB}$ ). Figure 5 shows the $\mathrm{S}_{11}$ and $\mathrm{S}_{21}$ simulation result. Transmission loss or known as S21 magnitude is a main parameters in all FSS analysis. It is used in measuring the transmission loss for the FSS, as it been known that the higher transmission loss the better FSS performance. A good FSS will produce the transmission loss less than $-10 \mathrm{~dB}$, which indicates that the FSS can blocks more than $90 \%$ of the certain frequency. While the return loss for the FSS are 0dB which are totally blocks the frequency from being transmit back. The simulation and the measured results are shown above in Figure 5 where both result for the simulation and measured are not approximately the same. For the measurement results its drops at $2.67 \mathrm{GHz}$, while the simulation results drops at $2.45 \mathrm{GHz}$. For the transmission loss the results for the measured are at $-60 \mathrm{~dB}$ while the simulation results are at $-65 \mathrm{~dB}$.

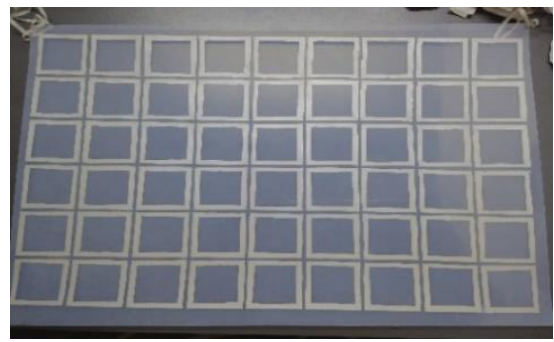

Figure 2. Fabricated FSS on flexible thin plastic substrate

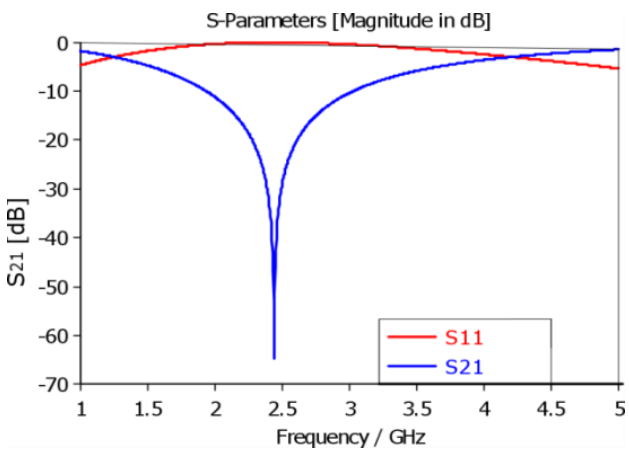

Figure 4. $S_{11}$ and $S_{21}$ simulation result

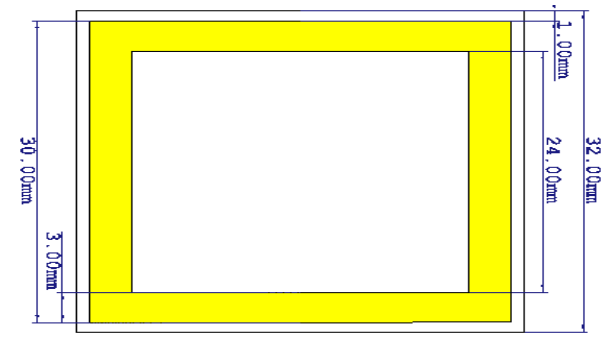

Figure 3. FSS square elements shape design

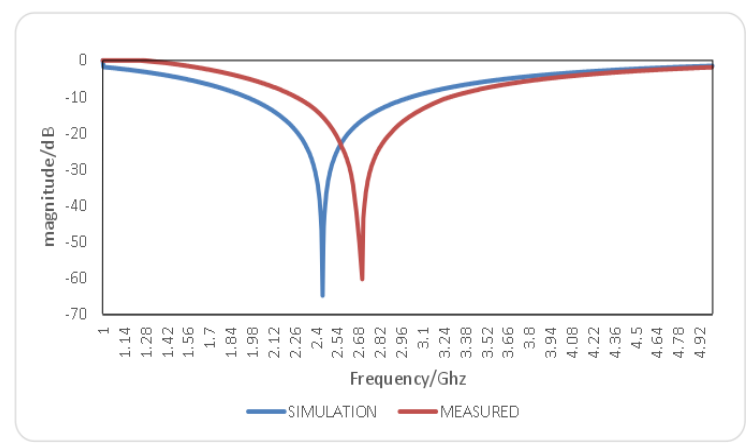

Figure 5. Simulation and measured results

The measured frequency are shifted by $9 \%$ to higher frequency, but the frequency drop for the bandstop are begin at $-10 \mathrm{~dB}$ so it still can be accepted. This is because of the length or size of the fabricated FSS are smaller than actual size in the simulated FSS. This is because of the fabrication process are being measured manually one by one for the length or size of the square for the one sheet of the flexible substrate. The square FSS exhibits the band-stop frequency response that resonates at the frequency of $f=1 /(2 \pi \sqrt{ } \mathrm{LC})$ where $\mathrm{L}$ are the inductance and $\mathrm{C}$ are the capacitance. The value of the LC are based on the size of the conductor. So by changing the size or length of the conductor the center of the band-stop also will be shifted respectively. The change in the conductor elements will change the behavior of the frequency response characteristic based on the equivalent circuit.

\section{PARAMETRIC ANALYSIS}

This project paper also consider about the different of unit cell size. According to the initial design, the unit cell size that was used for this design is $16 \mathrm{~mm}$ were shown in Figure 6 . As the height of the initial design is constant which is the height of the flexible thin plastic substrate is $0.5 \mathrm{~mm}$, this section were covers only about the different of the unit cell size from $14 \mathrm{~mm}$ up to $18 \mathrm{~mm}$. With the parameters analysis for the different size of the units cell, it can be proof that the frequency drop are effected by the size of the unit cell size just like been shown in the Figure 6 . When optimized the unit cell size it also simultaneously effect the gap space between the two conductor elements for the FSS. The gap space between two conductors of the 
FSS are shown in Figure 7. The parameters analysis will change and does not maintain the gap space as the gap space between two conductors are depends by the unit cell size of the square shape itself. The parameters analysis that does not eliminated the gap space between two conductor are at $16 \mathrm{~mm}, 17 \mathrm{~mm}$ and $18 \mathrm{~mm}$, while the other two parameter analysis which are $15 \mathrm{~mm}$ and $14 \mathrm{~mm}$ will eliminate the gap space and may overlap with the length of the outer square.

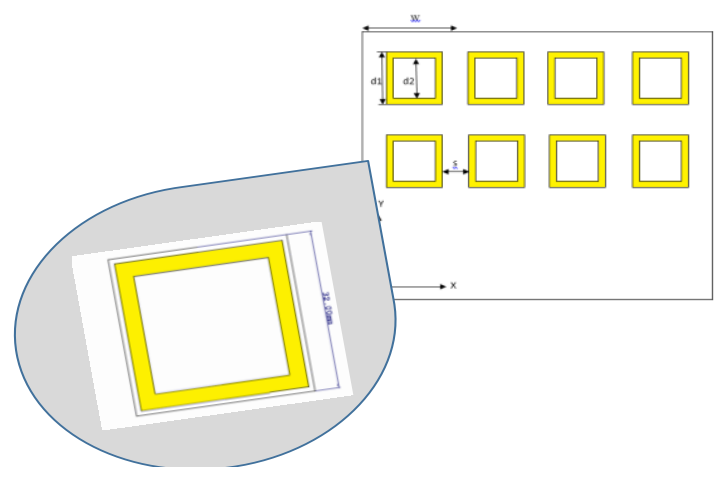

Figure 6. Unit cell size of FSS

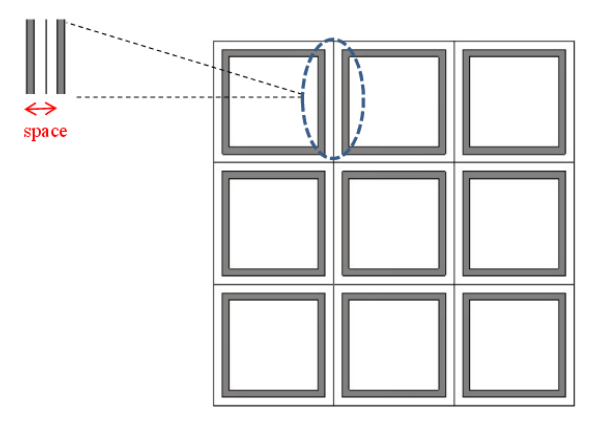

Figure 7. Gap space on the FSS

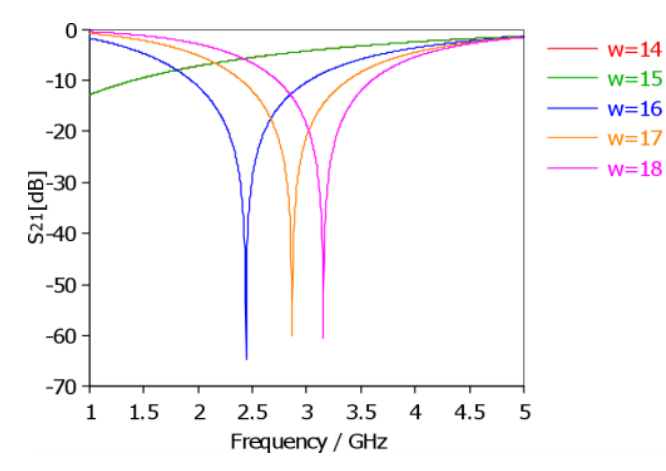

Figure 8. $\mathrm{S}_{21}$ result for different unit cell size from $14 \mathrm{~mm}$ to $18 \mathrm{~mm}$

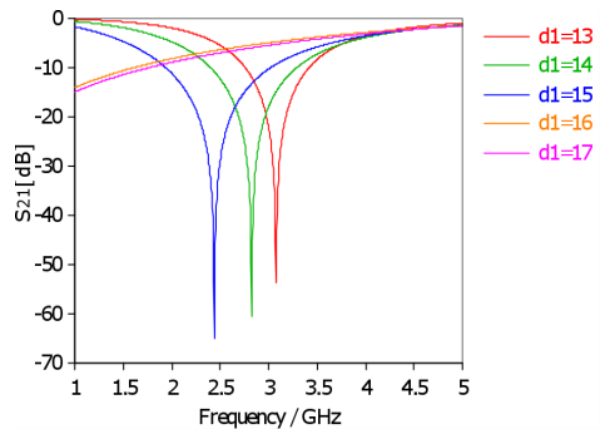

Figure 9. $\mathrm{S}_{21}$ result for different length of outer square from $13 \mathrm{~mm}$ to $17 \mathrm{~mm}$

Figure 8 shows how the size of the unit cell effects the frequency and the transmission of the FSS. The unit cell size that been used for this design are $16 \mathrm{~mm}$. The graph shows that when the size of the unit cell change to smaller value such as $14 \mathrm{~mm}$ and $15 \mathrm{~mm}$, the frequency and the transmission value cannot be determined or read as the frequency drop for that unit cell size is out from the frequency range. This is due to the length of the outer square and the unit cell are on the same size as mention before. So from the parametric study, the unit cell size of the FSS should be bigger than the length of the outer square that being used. From the graphs also its shows that if the bigger size of the unit cell size such as $17 \mathrm{~mm}$ and $18 \mathrm{~mm}$ being used the frequency also will be shifted to bigger frequency. From the graph in Figure 8, it can be conclude that at $16 \mathrm{~mm}$ size of the unit cell for the FSS can be seen to cycle at the best performance at the particular frequency with the best transmission loss. The separation between two elements of the unit cell is preferred that an appropriate gap is a must and conformity of the dimension of the unit cell is needed to obtain a good results.

Aside from the difference of the unit cell size, the difference of the length of the outer square also being considered in this project. According to the initial design, the length of outer square that was used for this design is $15 \mathrm{~mm}$. As the height of the initial design is constant which is the height of the copper is $0.035 \mathrm{~mm}$, this section were covers only about the different of the length of the outer square from $13 \mathrm{~mm}$ up to $17 \mathrm{~mm}$. The length of the outer square of the FSS was changed in several parameters value in order to studies the relationship between the FSS design and the performance of the frequency for the design FSS as shown in the Figure 9. Figure 9 shows how the length of the outer square effects the frequency and the transmission of the FSS. The length of the outer square that being used for this design are $15 \mathrm{~mm}$. The graph shows that the biggest value such as $16 \mathrm{~mm}$ and $17 \mathrm{~mm}$ they are not suitable to be used for the length of the outer square as 
its overlap with the unit cell size. As the results the frequency and the transmission cannot be determined as it when out of frequency range. For the length of smaller value it's also not suitable to be used as it's shown in the graph that the frequency will be shifted to bigger frequency. It is inversely proportional to each other. This can be verified by the basic frequency formula as the wavelength of the FSS is decreases the frequency will be increase.

Another parameters that being considered in this project are the difference of the length of the inner square. The length of the inner square of the FSS are also as important of the length of the outer square of the FSS. As it's also effects the frequency shift and the transmission of the FSS. Based on the initial design of the FSS, the length of the inner Square is $12 \mathrm{~mm}$. As the height of the initial design is remain constant which is the height of the copper is $0.035 \mathrm{~mm}$, this section were covers only about the different of the length of inner square from $10 \mathrm{~mm}$ up to $14 \mathrm{~mm}$. From the parameters study for the length of the outer and inner square of the FSS the width of the conductor can be determined from the different of the length. Figure 10 shows the width of the conductor.

Figure 11 shows how the frequency and the transmission of FSS are effected by the length of the inner square shape. The length of the outer square that being used for this design are $12 \mathrm{~mm}$. From the graph it can be seen that the higher value of the length of the inner square being used the frequency will be shifted to lower frequency. Meanwhile, the lower value of the length of the inner square being used the higher value of the transmission of the FSS and the higher the frequency. As we know that the higher the transmission loss, the better FSS we have. But it is also important to consider the frequency value for the FSS. So from graph the best value that can be used for the length of the inner square are $12 \mathrm{~mm}$. Aside from the unit cell size and the length of the square, the other characteristic of the FSS are also being monitored. One of the characteristic is the electrical field of the FSS or known as E-field. Coulomb's Law states that the electrical fields are produce from the electrical charges and the changing current of the electrical field will create a magnetic field this are stated by the Ampere's Law but Faraday's law also stated that a changing magnetic field can create an electric field. The E-field and the H-field of the FSS that are produced can be seen in the Figure 12.

Figure 12 shows the direction of the arrows for the E-filed and the H-field, it's the same with the direction of the flaming right hand rule. For the E-field the direction of the arrows are up and down, while for the $\mathrm{H}$-field are left and right. Just like what being stated by the Faraday and Ampere's law, the electric field will recreates of the magnetic field and the magnetic field also will recreates the electrical thus will propagates the electromagnetic waves. The electromagnetic wave are the combination of the magnetic and electric field that are vibrates together in space and time in a synchronous fashion. In a simple word, a looping electric field causes a looping magnetic field which will causes a looping electric field and vice versa and the electromagnetic wave also propagates effectively and will produce a pattern or shape of the electromagnetic wave like the perpetual game of the leapfrog. The function of the $\mathrm{E}$ and $\mathrm{H}$ can be proved if it is substitutes into the Maxwell's equation. The complete cycle of the E and H also play an important rule as its will determine the wavelength and the frequency of the FSS can be determines just like the basic formula of the wavelength and the frequency. The E-field and H-field not only can propagates the electromagnetic waves but it also can produces the surfaces current of the FSS. This is because the changing magnetic field of the FSS can generate a current although it wasn't being supply with electrical supply at all. Figure 13 will show the surfaces current of the FSS.

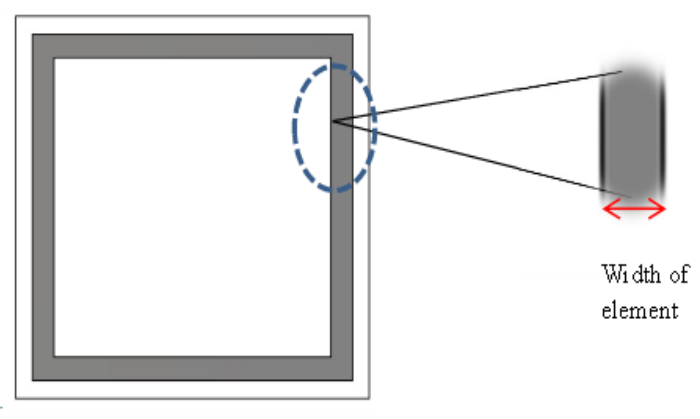

Figure 10. The width of the conductor

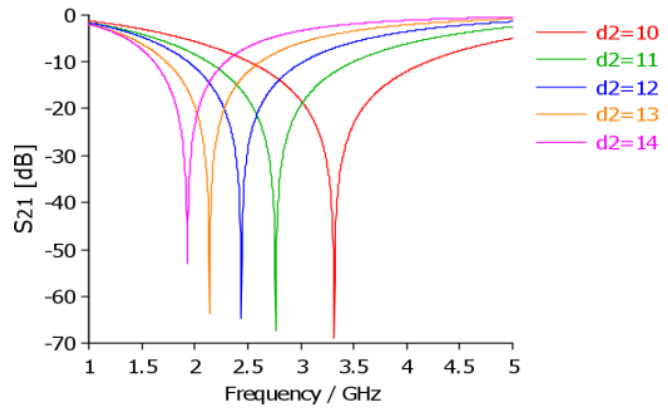

Figure 11. $\mathrm{S}_{21}$ result for different length of Inner Square from $10 \mathrm{~mm}$ to $14 \mathrm{~mm}$ 


\section{CONCLUSION}

Frequency Selective Surfaces (FSS) are planar of periodic structures that behave like a filters to electromagnetic energy. FSS are usually formed by a periodic array of a radiating elements. Generally FSS is a 2D planar structure consisting of one or more metallic patterns, each of it backed by a dielectric substrate. These structures are usually arranged in a periodic fashion and their frequency response is entirely determined by the geometry of the structure in one period called a unit cell. Different elements shape of FSS offer different frequency response. The square elements shape as its gives the best performance in frequency characteristic and has simple shape for the fabrication process, FSS on the flexible thin plastic substrate has shown a superior performance then the current FSS.

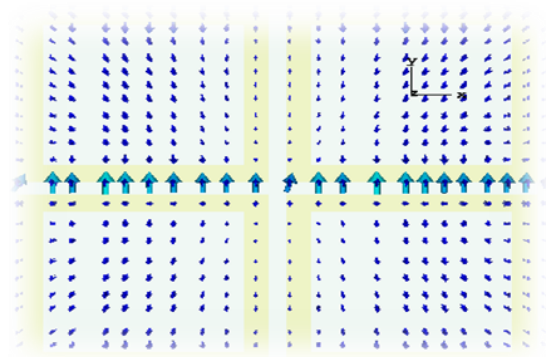

(a) E-field of the FSS

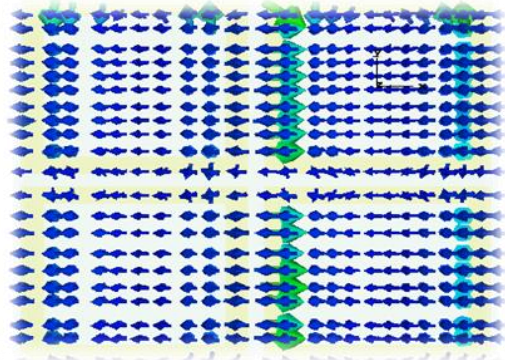

(b) H-field of the FSS

Figure 12. The direction of the arrows for the a) E-field and b) H-field of the FSS

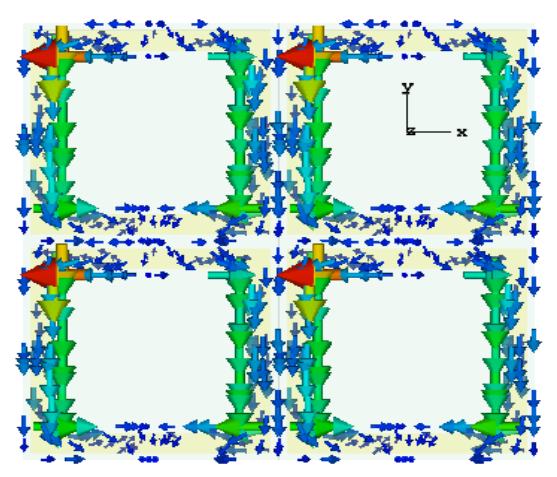

Figure 13. Surface current of FSS

\section{ACKNOWLEDGEMENTS}

The authors gratefully acknowledge use of the services and facilities of the Advanced Communication Engineering Centre (ACE) CoE, School of Computer and Communication Engineering, Universiti Malaysia Perlis (UniMAP). This project also been funded by Fundamental Research Grant Scheme (FRGS) 9003-00545.

\section{REFERENCES}

[1] Munk, B.A., "Frequency selective surfaces: theory and design". Wiley-Interscience. 2005

[2] V. Kottapalli, A. Kiremidjian, J. Lynch, E. Carryer, T. Kenny, K. Law and Y. Lei, "Two-tiered wireless sensor network architecture for structural health monitoring", in SPIE's 10th Annual International Symposium on Smart Structures and Materials, San Diego,2003.

[3] Fallahi, A., Mishrikey, M., Hafner, C., \& Vahldieck, R. "Efficient procedures for the optimization of frequency selective surfaces". Antennas and propagation, IEEE Transactions on, 56(5), 1340-1349, 2008.

[4] A. Araujo, J. Garcia-Palacios, J. Blesa, F. Tirado, E. Romero, A. Samartin and O. Nieto-Taladriz, "Wireless Measurement System for Structural Health Monitoring With High Time-Synchronization Accuracy", IEEE Transactions on Instrumentation and Measurement, 61, no. 3, pp. 801-810, 2012.

[5] S.N Azemi, K. Ghorbani, K., and W.S.T Rowe, "A reconfigurable FSS using a spring resonator element”, IEEE Antennas and Wireless Propagation Letters, 12, 781-784, 2013. 
[6] P. Kala Monica and M. Susila, "Study of Frequency Selective Surface for Wireless Communication", Middle-East Journal of Scientific Research, 24, no. 5, pp. 1673-1678, 2016.

[7] Parker, Edward A., C. Antonopoulos, and N. E. Simpson. "Microwave band FSS in optically transparent conducting layers: performance of ring element arrays." Microwave and Optical Technology Letters 16, no. 2, 6163, 1997.

[8] Tsakonas, C., S. C. Liew, C. Mias, D. C. Koutsogeorgis, R. M. Ranson, W. M. Cranton, and M. Dudhia. "Optically transparent frequency selective window for microwave applications." Electronics Letters 37, no. 24,1464-1466, 2001.

[9] Han, Y., Y. M. Liu, P. Jin, B. Liu, J. Ma, and J. B. Tan. "Optical-transparent Wi-Fi bandpass mesh-coated frequency selective surface." Electronics Letters 50, no. 5, 381-383, 2014.

[10] Han, Yu, Yuxuan Liu, Lin Han, Jie Lin, and Peng Jin. "High-performance hierarchical graphene/metal-mesh film for optically transparent electromagnetic interference shielding." Carbon 115, 34-42, 2017.

[11] Azemi, S. N., Ghorbani, K., \& Rowe, W. S. "Development and analysis of 3D frequency selective surfaces." In Microwave Conference Proceedings (APMC), 2011 Asia-Pacific, pp. 693-696. IEEE, 2011.

[12] Azemi, S. N., Ghorbani, K., \& Rowe, W. S.. "Mechanically tunable and reconfigurable FSS using spring loaded ring resonators." In Proc. Eur. Microw. Conf., pp. 932-935. 2013.

[13] Ai, Jun, Huiqing Xiang, Qifeng Du, Dongchu Chen, Jianguo Liu, and Xiaoyan Zeng. "Rapid and high-performance processing technology for large-area frequency selective surfaces." Journal of Materials Processing Technology 264. 129-133, 2019.

[14] Azemi, S. N., Ghorbani, K., \& Rowe, W. S. "3D frequency selective surfaces with close band spacing". In Microwave Conference Proceedings (APMC), 2012 Asia-Pacific (pp. 454-456). IEEE.2012.

[15] Azemi, S.N., Ghorbani, K. and Rowe, W.S., 2012. 3D frequency selective surfaces. Progress In Electromagnetics Research, 29, pp.191-203, 2013.

[16] Pinheiro, G.J., de Oliveira, M.R., Silva Filho, H.V., de Melo, M.T., Neto, A.G. and de Sousa, T.R., 2019. Four-bit instantaneous frequency measurement systems based on frequency selective surfaces. Microwave and Optical Technology Letters, 61(1), pp.68-72, 2019. 